Article

\title{
Slope matched highly birefringent hybrid dispersion compensating fiber over telecommunication bands with low confinement loss
}

\author{
Amit Halder 1,* \\ ${ }^{1}$ Department of Electrical and Electronic Engineering, Rajshahi University of Engineering and Technology, \\ Rajshahi-6204; amit.rueten@ gmail.com \\ * Correspondence: amit.rueten@gmail.com;
}

\begin{abstract}
This article reveals a best possible design for hybrid dispersion compensating fiber (HyDCF) with high birefringence established on modified broadband compensating structure through $\mathrm{S}, \mathrm{C}$ and $\mathrm{L}$ telecommunication bands. The simulation outcome exhibits relatively higher birefringence of $3.76 \times 10^{-2}$ at wavelength of $1550 \mathrm{~nm}$. The suggested fiber also has dispersion compensation characteristics in an inclusive series of wavelengths which covers $1400-1625 \mathrm{~nm}$. The reported design can achieve dispersion quantity of $-606 \mathrm{ps} /(\mathrm{nm} . \mathrm{km})$ at $1550 \mathrm{~nm}$ effective wavelength. The reported fiber design matches the relative dispersion slope (RDS) $0.003694 \mathrm{~nm}^{-1}$ similar to single mode fiber at $1550 \mathrm{~nm}$ operating wavelength. This fiber demonstrates negatively flattened effective dispersion of $-2.703 \pm 0.734 \mathrm{ps} /$ (nm.km) within $180 \mathrm{~nm}$ flat band ranging from 1460-1640 nm wavelength. It is also convenient to optical high bit rate communication systems. The low confinement loss is found $3.756 \times 10^{-10} \mathrm{~dB} / \mathrm{m}$ at the operating wavelength. This design also achieves highly nonlinear coefficient of $50.34 \mathrm{~W}^{-1} \mathrm{~km}^{-1}$. In some cases, it can also be used in sensing applications.
\end{abstract}

Keywords: birefringence; confinement loss; photonic crystal fibre; relative dispersion slope; single mode operation

\section{Introduction}

Optical fiber waveguides are very suitable for fast data transmission in communication systems. The photonic crystal fibers are the type of optical fiber waveguide which consists of different periodic air hole arrangement in the silica base region [1]. Microstructure PCF designs are broadly used for various optical applications like sensing, dispersion compensation, ultrafast data transmission and nonlinearity. Dispersion property of optical fiber is a major problem in wavelength division multiplexing (WDM) and high bit rate transmission systems as it occurs pulse broadening and bandwidth limitation. In high speed WDM systems, compensation is necessary to the transmission characteristics like the dispersion and its slope of single mode fiber (SMF) [2]. Conventional fibers show high losses in data transmission systems too. Dispersion compensating fiber (DCF) with high negative dispersion is a good candidate to reduce the losses and costs which occurs in optical waveguides [3-7]. It is evident that for typical dispersion compensating fibers negative dispersion values varies from -100 to $-300 \mathrm{ps} /(\mathrm{nm} . \mathrm{km})$ at $1550 \mathrm{~nm}$ [8]. PCFs or holey fibers can be engineered to change the dispersion values in different wavelength parameters. The optical property like dispersion can be varied by changing the dimension of air-holes and their corresponding positions, [9-10] that is vital for dispersion compensation in a PCF design.

Birefringence is a property of maintaining optical polarization in fiber which is suitable for the optical sensing applications [30]. High birefringence can be achieved on a PCF structure when the asymmetrical shape is implemented in fiber core by engaging deliberate artificial defects at the centre core or the air holes in elliptical rather than circular shapes are made [11]. Achieving high birefringence the circular air holes were used rather than elliptical ones just to overcome the fabrication challenge. Elliptical air holes are difficult to implement in a microstructure or nanostructure designs.

In recent times, there are some proposal of PCF designs by researchers to encounter various optical 
characteristics like high birefringence and dispersion compensation. Such as, an octagonal shaped photonic crystal fiber recommended in [12] has encountered negative dispersion of - $588 \mathrm{ps} /(\mathrm{nm} . \mathrm{km})$ and the birefringence was reported $1.81 \times 10^{-2}$ at $1550 \mathrm{~nm}$. This design possess less than $10 \mathrm{~dB} / \mathrm{m}$ confinement loss. Kaijage et al. [13] proposed a modified octagonal PCF structure attaining the dispersion coefficient and birefringence $-239.5 \mathrm{ps} /(\mathrm{nm} . \mathrm{km})$ and $1.67 \times 10^{-2}$ respectively at $1550 \mathrm{~nm}$ operating wavelength. This modified PCF contains elliptical air holes near the centre core which has complexity in fabrication but has low confinement loss in the order of $10^{-5}$. In the past a golden spiral design was proposed which accomplished $1.60 \times 10^{-2}$ as its birefringence and $-400 \mathrm{ps} /(\mathrm{nm} . \mathrm{km})$ as its quantity of negative dispersion both observed at the same $1550 \mathrm{~nm}$ effective wavelength [14]. But confinement loss is not measured in this spiral structure because in previous spiral structures shows relatively high confinement losses. In 2016, a defected core PCF that consisted of four elliptical air holes near the centre core had been proposed which achieved birefringence of $3.373 \times 10^{-2}$ with negative dispersion of $-837.8 \mathrm{ps} /(\mathrm{nm} . \mathrm{km})$ over E, S, C and L communication bands in the same $1550 \mathrm{~nm}$ wavelength [15]. As it used elliptical air holes, some complexities were faced in fabrication of the fiber. Moreover, in some current works, $2.75 \times 10^{-2}$ birefringence and - $331 \mathrm{ps} /(\mathrm{nm} . \mathrm{km})$ amount of negative dispersion at $1550 \mathrm{~nm}$ operating wavelength through $\mathrm{S}+\mathrm{C}+\mathrm{L}$ bands has been offered [16] using a defected core circular PCF. A PCF design offered by Habib et al. [17] demonstrated negative dispersion varied from - 134 to $-385 \mathrm{ps} /(\mathrm{nm} . \mathrm{km})$ through $\mathrm{E}, \mathrm{S}, \mathrm{C}$, $\mathrm{L}$ and $\mathrm{U}$ wideband and showed birefringence $2.13 \times 10^{-2}$ at $1550 \mathrm{~nm}$ with relatively high confinement loss of $4.54 \mathrm{~dB} / \mathrm{m}$. In the same year a defected core decagonal PCF was also prescribed showing negative dispersion quantity of $-390 \mathrm{ps} /(\mathrm{nm} . \mathrm{km})$, has a very negligible birefringence in the order of $10^{-5}$ and confinement loss is about $3 \times 10^{-6} \mathrm{~dB} / \mathrm{m}$ at $1550 \mathrm{~nm}$ [18]. In the year 2016, Ali et al. proposed a hybrid PCF in [19] which reported birefringence of $2.78 \times 10^{-2}$ having a negative dispersion of $-345 \mathrm{ps} /(\mathrm{nm} . \mathrm{km})$ where an asymmetric core was created on a circular based cladding. This structure maintains confinement loss is about less than $10^{-2} \mathrm{~dB} / \mathrm{m}$ at effective wavelength of $1.55 \mu \mathrm{m}$.

In this article, we offer a hybrid dispersion compensating PCF (HyDCF) that is very satisfactory for compensating the dispersion of single mode fiber over the S, C and L communication bands. The reported fiber also acquired birefringence of $3.76 \times 10^{-2}$ along with $-606 \mathrm{ps} /(\mathrm{nm} . \mathrm{km})$ negative dispersion at $1550 \mathrm{~nm}$ operating wavelength over the S, C and L communication bands. The residual dispersion slope was matched to $0.003694 \mathrm{~nm}^{-1}$ with respect to single mode fiber. The nonlinear property of this proposed HyDCF was also observed and its value is $50.34 \mathrm{~W}^{-1} \mathrm{~km}^{-1}$ at $1550 \mathrm{~nm}$. As the proposed design shows the single mode property from 1350-1700 $\mathrm{nm}$ wavelength and so on, it may be very beneficial for high bit stream rate fast transmission over the long distance of an optical fiber communication channel. As it exhibits the dual property, the suggested fiber is a best nominee for both sensing \& telecommunication applications effectively. This fiber also demonstrates confinement loss in order of $10^{-10}$ which is comparatively lower than some hybrid dispersion compensating PCF structures.

\section{Materials and Methodology}

The designed microstructure of HyDCF was based on a pure fused silica material. The circular air hole cladding consists of hexagonal inner structure with octagonal outer structure. Circular air holes structured in a periodic order has been used in the fibre cladding. Figure 1 displays the transverse cross-sectional view of the proposed fibre design. The artificial defection in core was made with six air hole rings in the cladding comprised of two hexagonal rings in inner cladding layer and four octagonal rings in outer cladding layer to construct the hybrid PCF. The inner core was made asymmetrical by reducing the diameter of two air holes among the six air holes in the first ring.

Four octagonal circular air hole rings were used in the outer cladding to minimize the confinement loss of the fibre and the defected inner core was introduced to achieve high birefringence. The diameter of smaller air holes was $\mathrm{dc}=0.24 \mu \mathrm{m}$. First and second ring air hole diameter of the hexagonal structure was $d_{1}=d_{2}=0.74$ $\mu \mathrm{m}$. The air holes from the third to sixth of the octagonal structure was $d=0.52 \mu \mathrm{m}$. The two smaller air holes in the first ring were adjusted to attain high birefringence with negative dispersion solidity as this has an impact over these optical characteristics. Air-hole to air-hole distance of two consecutive rings is recognized as the pitch $(\Lambda)$ of the structure. 


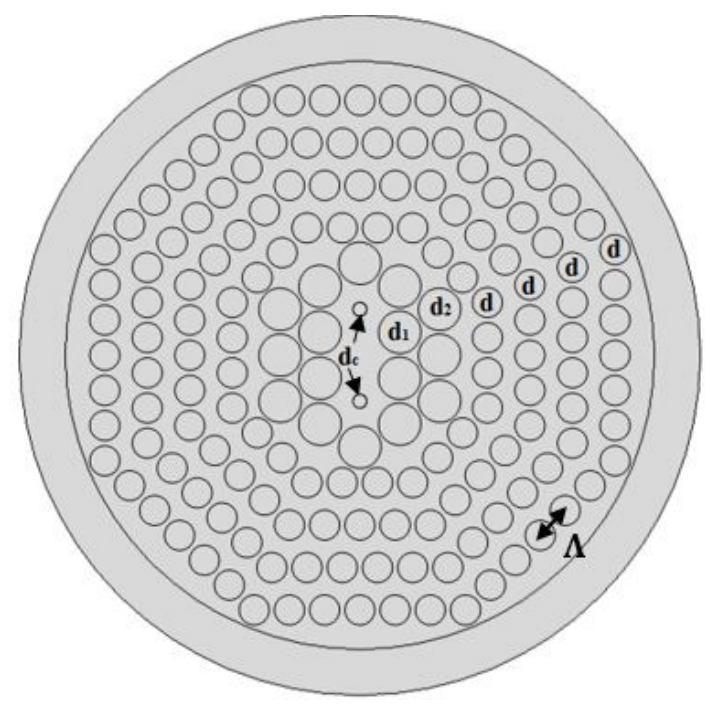

Figure 1. Cross sectional view of proposed hybrid PCF design. Where, pitch, $\Lambda=0.8 \mu \mathrm{m}, \mathrm{d}_{\mathrm{c}}=0.24 \mu \mathrm{m}$, $\mathrm{d}_{1}=\mathrm{d}_{2}=0.74 \mu \mathrm{m}$ and $\mathrm{d}=0.52 \mu \mathrm{m}$.

In this structure pitch was taken $0.8 \mu \mathrm{m}$ as optimum which made this design proper for practical fabrication process as it is closer to $1 \mu \mathrm{m}$. There is a significant development of fabrication process in recent times, specifically drilling, sol-gel casting, tapering, and lithography [20, 21] without any major difficulty it is possible to draw our HyDCF structure. In recent times, Sol-gel technique is used to reduce the fabrication challenges of asymmetrical dimensions and positions of air hole construct on a silica base in PCF designs [22]. Wiederhecker et al. [33] fruitfully implement standard stack-and-draw technique, and have a high air-filling fraction cladding and a tube-like core at nanoscale as $110 \mathrm{~nm}$ and $200 \mathrm{~nm}$. The complex proposed PCF structures like Arif et al. [35] and Ahmed et al. [36] also suggested Sol-gel technique is a better technique to fabricate this type of structures. In this structure hybrid cladding was presented and there were two small air holes closer to the core region which was problematic to fabricate in stack and draw method. But Ruan et al. [34] successfully fabricated as small as $20 \mathrm{~nm}$ air hole in fiber core by using extrusion technique. So, both sol-gel and extrusion technique is a better way to fabricate the proposed PCF structure.

\section{Simulation Techniques and Equations}

Finite element method (FEM) has been applied to exemplify the performance parameters of the HyDCF. A commercial full vectorial software has been implemented to simulate and calculate the modal characteristics of the proposed fibre. Cross sections of PCF designs with a static number of air- holes are separated into consistent subspaces where Maxwell's equations are solved by FEM method for the neighbouring subspaces. The subspaces are triangular in shape and it provides a good approximation in PCF structures. Following vectorial equation is obtained from Maxwell's curl equations of electromagnetics [23].

$$
\nabla \times\left([s]^{-1} \nabla \times \vec{E}\right)-k_{0}^{2} n_{e f f}^{2}[s] \vec{E}=0
$$

Here, electric field vector and free-space wave number is denoted by $\vec{E}$ and $k_{0}$ respectively, operating wavelength is $k$, effective refractive index is $n_{e f f}=\beta / k_{0}$, where, $\beta$ is propagation constant. Free-space wave number can be computed by this equation $k_{0}=2 \pi \lambda$. In this computation process Sellmeier equation was used to calculate the effective refractive index of the fiber in different wavelengths which leads to compute the dispersion, confinement loss and normalize frequency. The equation is as follows 


$$
n(\lambda)=\sqrt{1+\frac{B_{1} \lambda^{2}}{\lambda^{2}-C_{1}}+\frac{B_{2} \lambda^{2}}{\lambda^{2}-C_{2}}+\frac{B_{3} \lambda^{2}}{\lambda^{2}-C_{3}}}
$$

where, $\mathrm{n}$ and $\lambda$ represent the refractive index and the wavelength respectively; $\mathrm{B}_{1}, \mathrm{~B}_{2}, \mathrm{~B}_{3}, \mathrm{C}_{1}, \mathrm{C}_{2}, \mathrm{C}_{3}$ are the coefficient of fiber background material silica.

Circular perfectly matched layer (PML) boundary is used in the FEM method to simulate the wavelength dependence PCF optical properties. FEM solves the Maxwell equations directly in best approximation to acquire the value of the effective refractive index, $n_{\text {eff. }}$ Once, is computed for each wavelength, the dispersion $D$, birefringence $B$, effective area $A_{\text {eff }}$ and confinement loss $L_{c}$ can be calculated through the following equations.

$$
\begin{gathered}
D(\lambda)=-(\lambda / c) \frac{d^{2}\left(\operatorname{Re}\left[n_{e f f}\right]\right)}{d \lambda^{2}} \\
L_{c}=8.686 \times k_{0} \operatorname{Im}\left[n_{e f f}\right] \\
B=\left|n_{x}-n_{y}\right|
\end{gathered}
$$

where, $R e\left[n_{e f f}\right]$ and $\operatorname{Im}\left[n_{\text {eff }}\right]$ are the real and imaginary parts of the effective refractive index $n_{\text {eff }}$ respectively; $\lambda$ is the space wavelength, velocity of light in vacuum is c and $k_{0}$ is the free-space number.

The effective area $A_{\text {eff }}$ can be calculated as follows [32]:

$$
A_{e f f}=\left(\iint|\vec{E}|^{2} d x d y\right)^{2} / \iint|\vec{E}|^{4} d x d y
$$

where, $A_{\text {eff }}$ is measured in $\mu \mathrm{m}^{2}$ and $\vec{E}$ is electric field in the medium. Therefore, nonlinear phenomena in PCFs can be studied by effective area too.

As single mode fibre shows positive dispersion and dispersion slope, the requirements for a DCF should be large negative dispersion and a dispersion slope over a broad series of wavelengths. The total residual dispersion of the fibre is computed by the following equation [26]:

$$
D_{T}=D_{S M F} \cdot L_{S M F}+D_{D C F} \cdot L_{D C F}
$$

where, $D_{S M F}$ and $D_{D C F}$ are denoted as the dispersion coefficients of the SMFs and DCFs, respectively. If it is required to compensate the total dispersion, the length of DCFs $\left(L_{D C F}\right)$ should be like that it makes the residual dispersion zero. Also the matching of dispersion slope as same as single mode fibre is needed. The residual dispersion slope can be calculated by the following equation [27]:

$$
S_{\text {slope }}=S_{S M F} \cdot L_{S M F}+S_{D C F} \cdot L_{D C F}
$$

where, $S_{S M F}$ and $S_{D C F}$ are the dispersion coefficients of the single mode fiber and dispersion compensating fiber, respectively.

Deliberately investigating the mode property of the proposed HyDCF it was seen that single mode of the fibre was varied according to effective $\mathrm{V}$ parameter inside the telecommunication band. Normalize frequency $V_{\text {eff }}$ factor for the HyDCF can be computed by using the ensuing equation [28]:

$$
V_{\text {eff }}=\frac{2 \pi \Lambda}{\lambda} \sqrt{n_{\text {core }}^{2}-n_{c l}^{2}} \geq 2.405
$$

Eq. (9) is executed to authenticate the single mode behavior of the proposed structure where represents the wave number in the free space and $\Lambda$ is pitch; $n_{\text {core }}$ and $n_{c l}$ denote the effective refractive index of core and cladding, correspondingly computed by Sellmeier equation and mode analysis by finite element method. The cut-off frequency of the effective $\mathrm{V}$ parameter is 2.405 for single mode fibres [29].

The numerical calculations like chromatic dispersion, birefringence, effective area, confinement loss, total residual dispersion, residual dispersion slope and V parameter are accomplished by Matlab software with the assistance of equations related to each term stated above.

\section{Simulation Results and Discussions}

Chromatic dispersion characteristics of both $\mathrm{x}$ and $\mathrm{y}$ polarization for optimum design parameters with 
$\mathrm{d} / \Lambda=0.65, \mathrm{~d}_{\mathrm{c}} / \Lambda=0.3, \mathrm{~d}_{1} / \Lambda=\mathrm{d}_{2} / \Lambda=0.92$ and pitch $\Lambda=0.8 \mu \mathrm{m}$ is shown in figure 2 (a). The global diameter of the smaller air holes of the 1 st ring of the hexagonal cladding structure is $d_{c} / \Lambda=0.3$ and other air holes of the 1 st and 2 nd rings are $d_{1} / \Lambda=d_{2} / \Lambda=0.92$. The octagonal ring structure from the 3 rd to the 6 th air hole rings have the diameter parameter of $d / \Lambda=0.65$. To reduce the complexity of fabrication the adjacent air holes are separated to a nominal distance. The plot of the wavelength versus dispersion shows the proposed HyDCF has negative dispersion coefficient about - 193.9 and $-606 \mathrm{ps} /(\mathrm{nm} . \mathrm{km})$ along the $\mathrm{x}$ and y polarization respectively at $1550 \mathrm{~nm}$. The y polarization dispersion is considered as the optimum value of dispersion in this work. Due to high negative dispersion, the proposed HyDCF could be an appropriate candidate for dispersion compensating in high bit rate transmission system. The property of the proposed fiber is affected by the diameter pitch as a function of wavelength for this reason the pitch value has been varied to observe the deviations in dispersion and birefringence. The effect by varying global diameter of pitch $0.8,0.9,1$ and $1.1 \mu \mathrm{m}$ while the other parameters are kept constant is revealed by figure 2(b). When the value of pitch is varied $0.8,0.9,1$ and $1.1 \mu \mathrm{m}$ their corresponding y polarization dispersion value becomes $-606,-277,-116.7$ and $-27.52 \mathrm{ps} /(\mathrm{nm} . \mathrm{km})$, respectively.

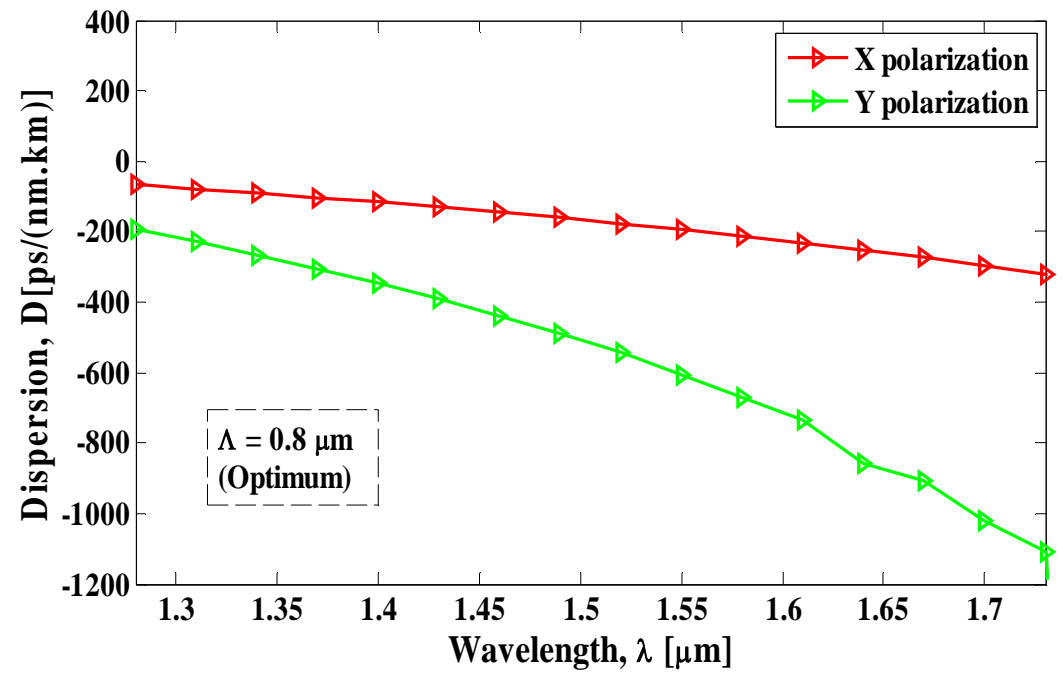

(a)

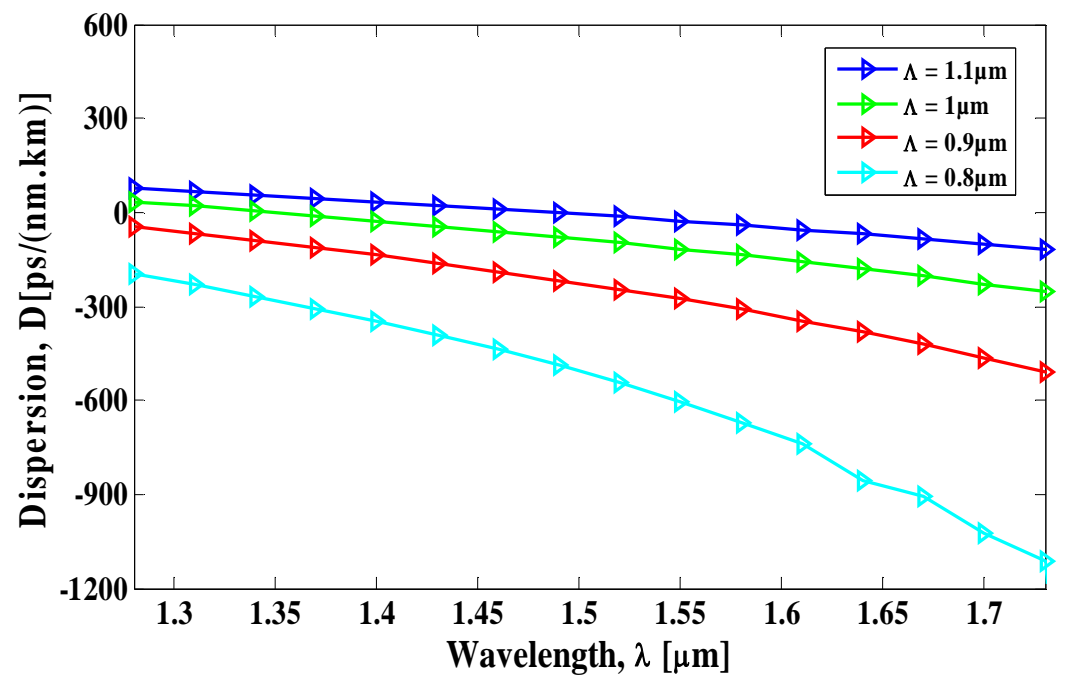

(b)

Figure 2. Dispersion as a function of wavelength curve for (a) both $x$ and y polarization. (b) Wavelength versus $y$ polarization dispersion in different pitch values. 
Birefringence characteristics of the proposed HyDCF are shown in figure 3. This particular property of the proposed HyDCF design will provide sensing applications [24]. The asymmetrical design of the proposed fiber core design reveals high birefringence, which is necessary for polarization maintaining applications. However, existing conventional PM fibers show a modal birefringence of about $5 \times 10^{-4}[25]$. Moreover, our proposed PCF demonstrates birefringence of about $3.76 \times 10^{-2}$ in optimum design parameters, which could be a proper contender in sensing applications. In figure 3, the effect of pitch on birefringence is also measured. As the value of pitch was taken $0.8,0.9,1$ and $1.1 \mu \mathrm{m}$ the birefringence at $1550 \mathrm{~nm}$ in this value becomes $0.03755,0.03257,0.02767$, and 0.02336 respectively. It exhibits the inverse relationship of birefringence and pitch of the HyDCF.

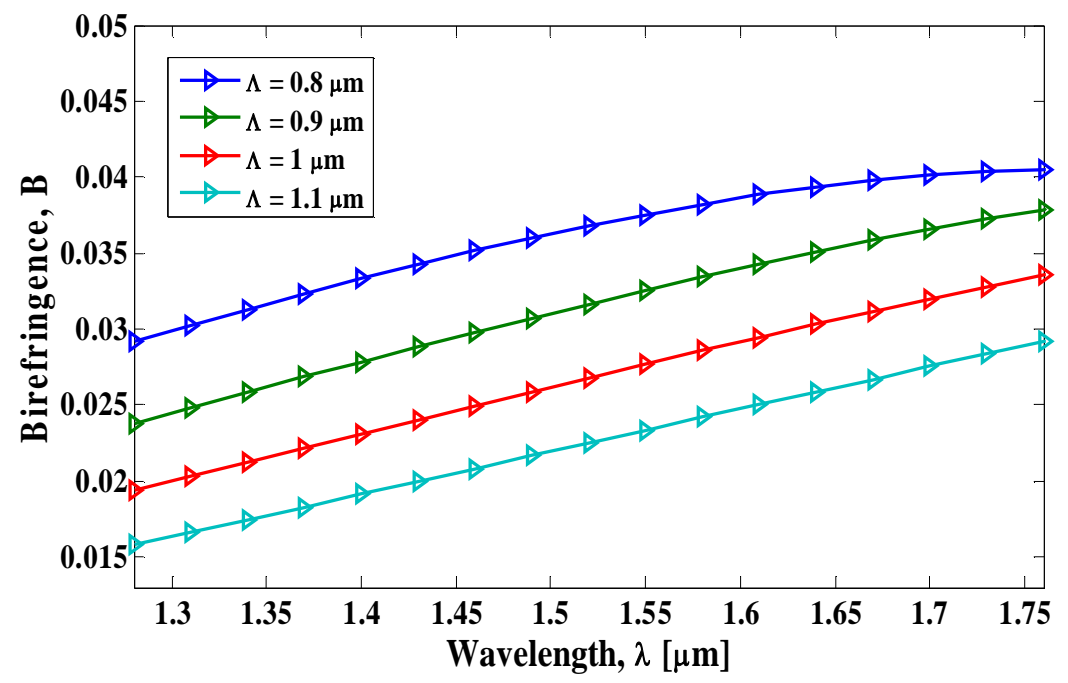

Figure 3. Wavelength versus birefringence plot for optimum design parameters and different pitch values of HyDCF.

The reported microstructure HyDCF reveals single mode operation over the band of interest starting from $1350 \mathrm{~nm}$ to $1800 \mathrm{~nm}$ in figure 4. The proposed HyDCF shows that at $1550 \mathrm{~nm}$ operating wavelength the value of $\mathrm{V}$ parameter is 2.282 (i.e. $V_{e f f}<2.405$ ) which fulfils the criteria to be a single mode fibre. It is also evident that the fibre demonstrates endlessly single mode operation through 1350-1800 nm wavelengths covering E, S, C, L and U telecommunication bands.

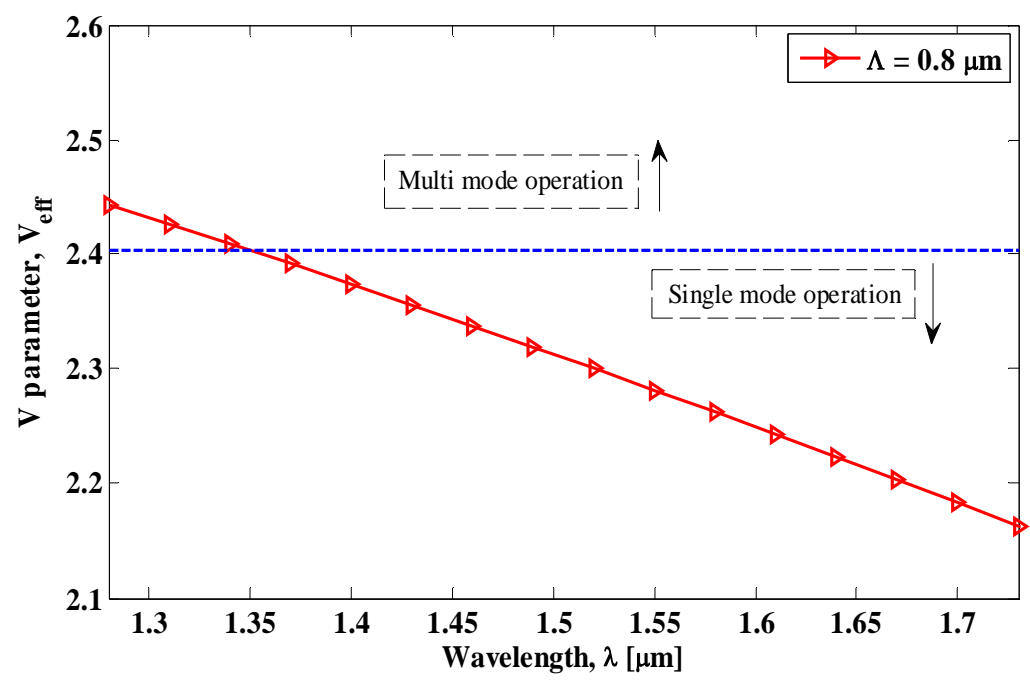

Figure 4. Wavelength dependence V parameter curve of the proposed HyDCF for optimized parameters. 
From the analysis of figure 5(a) it is evident that the RDS value of our proposed HyDCFs is 0.003694 $\mathrm{nm}^{-1}$ which matches to the single mode fibres' RDS slope. To attain better dispersion compensation, it is necessary to match the RDS of single mode fiber. Effective dispersion curve of the proposed HyDCF shown in figure 5(b) is negative dispersion flattened and the value of flattened dispersion coefficient is of -2.703 $\pm 0.734 \mathrm{ps} /$ (nm.km) within $180 \mathrm{~nm}$ flat band ranging from $1460 \mathrm{~nm}$ to $1640 \mathrm{~nm}$ wavelength. Compensation ratio (CR) of dispersion of fiber is also analyzed in figure 5(c) to prove the accomplishment of negative flattened dispersion at the range of $1460 \mathrm{~nm}$ to $1640 \mathrm{~nm}$ wavelength. After compensating of dispersion through $1 \mathrm{~km}$ long suggested HyDCF it is found to be equal to the total dispersion in $50 \mathrm{~km}$ long SMF, the residual dispersion versus wavelength plot is shown in figure 5(d). The value of residual dispersion is negatively flatten at the range of $1460 \mathrm{~nm}$ to $1640 \mathrm{~nm}$ wavelength band. Analyzing the results, it is evident that this HyDCF design could be an appropriate nominee for high bit-rate transmission systems over the band of interests.

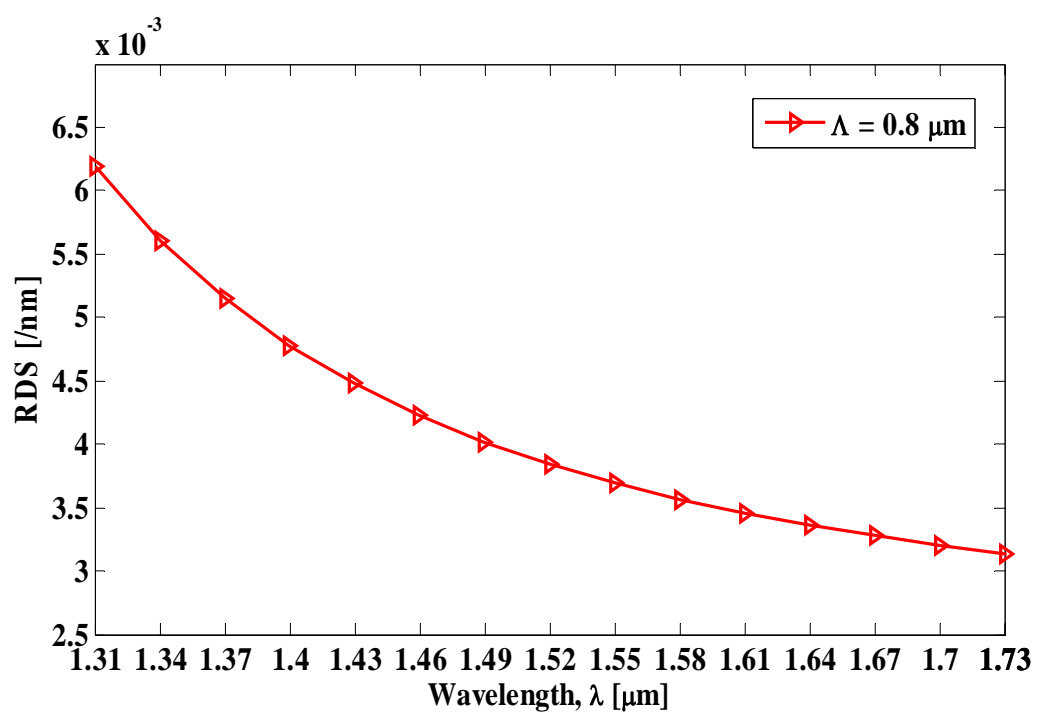

(a)

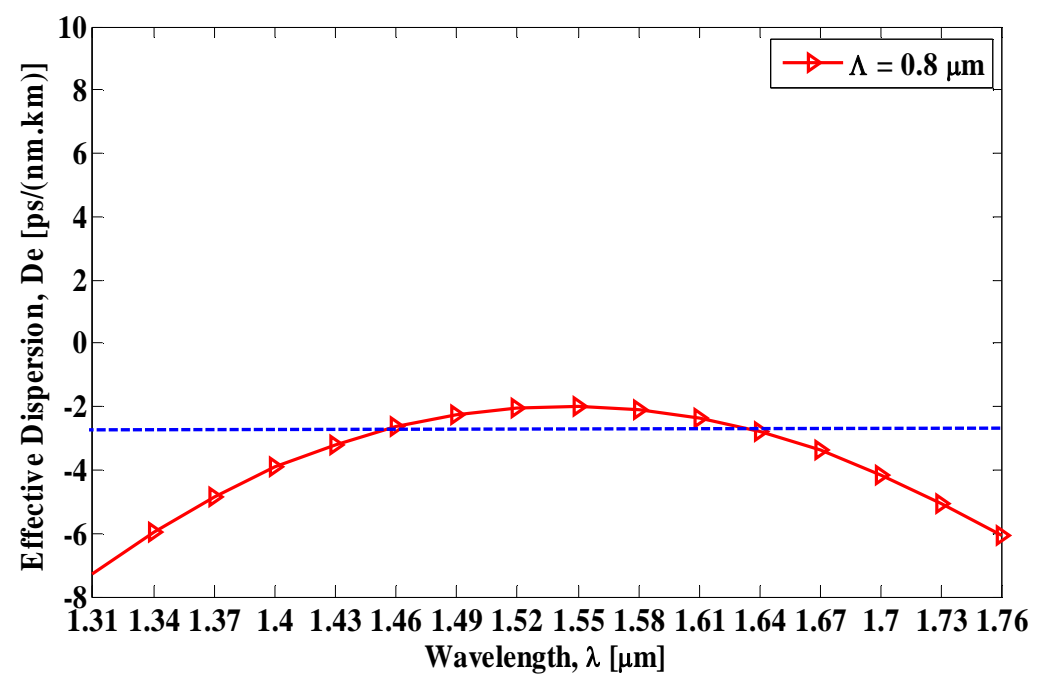

(b) 


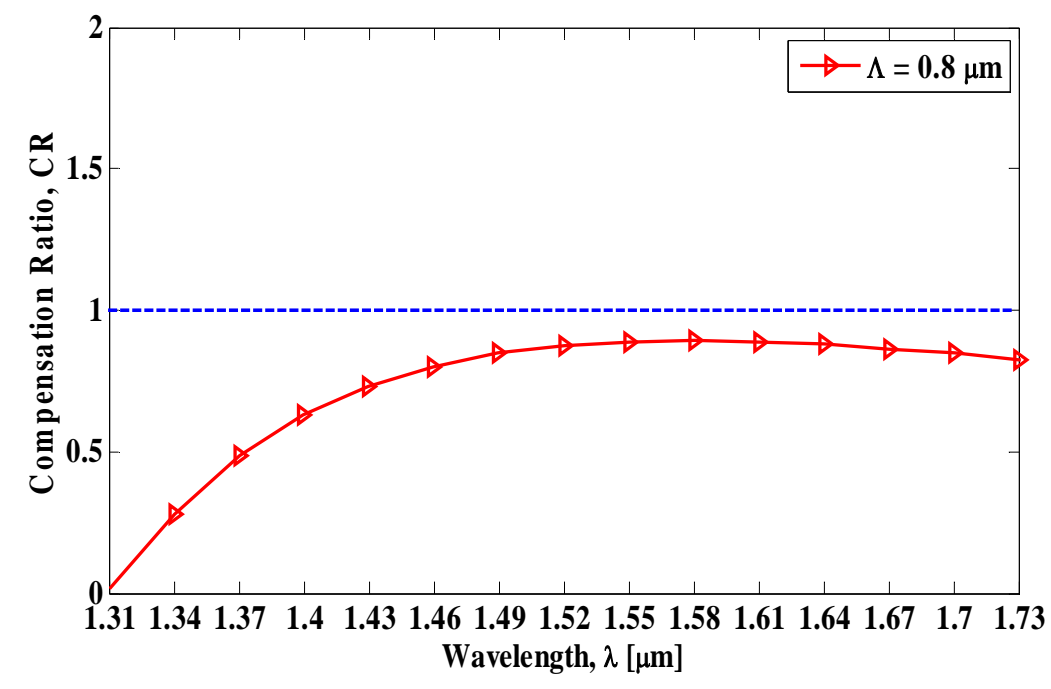

(c)

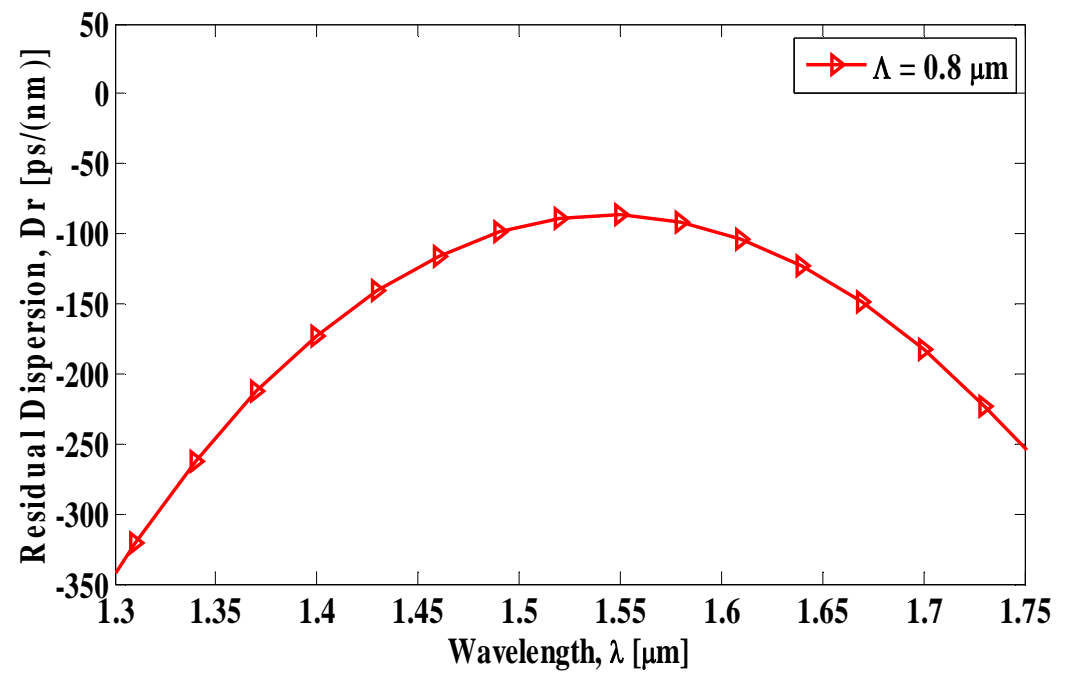

(d)

Figure 5. Wavelength dependent (a) Relative dispersion slope curve, (b) Effective Dispersion curve, (c)

Compensation Ratio (CR) curve and (d) Residual Dispersion curve of the proposed HyDCF after compensating for

$50 \mathrm{~km}$ long SMFs.

It is perceived in figure 6(a) that the optimal value of effective area of the HyDCF is $2.327 \mu \mathrm{m}^{2}$ at $1550 \mathrm{~nm}$ wavelength. The nonlinearity plot of the reported HyDCF for optimum pitch of the design parameters is observed in figure 6(b). The measure of nonlinearity at optimum pitch value is $50.34 \mathrm{~W}^{-1} \mathrm{~km}^{-1}$ at $1550 \mathrm{~nm}$ operating wavelength. According to the results demonstrated, the suggested design of HyDCF contains decent effective area and nonlinearity for the use of nonlinear applications. 


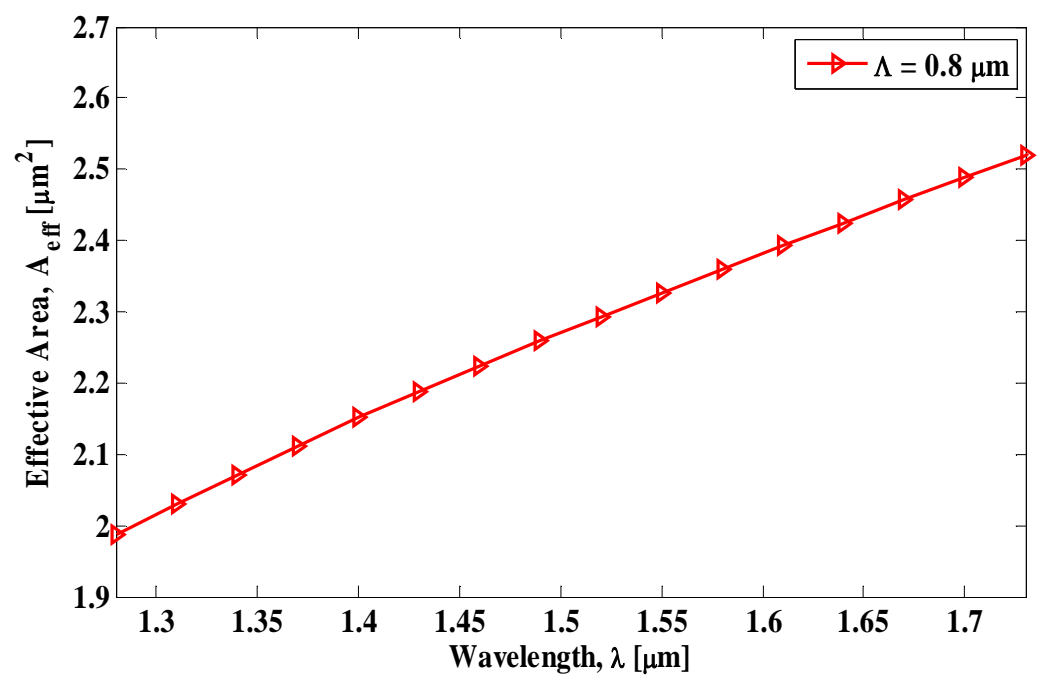

(a)

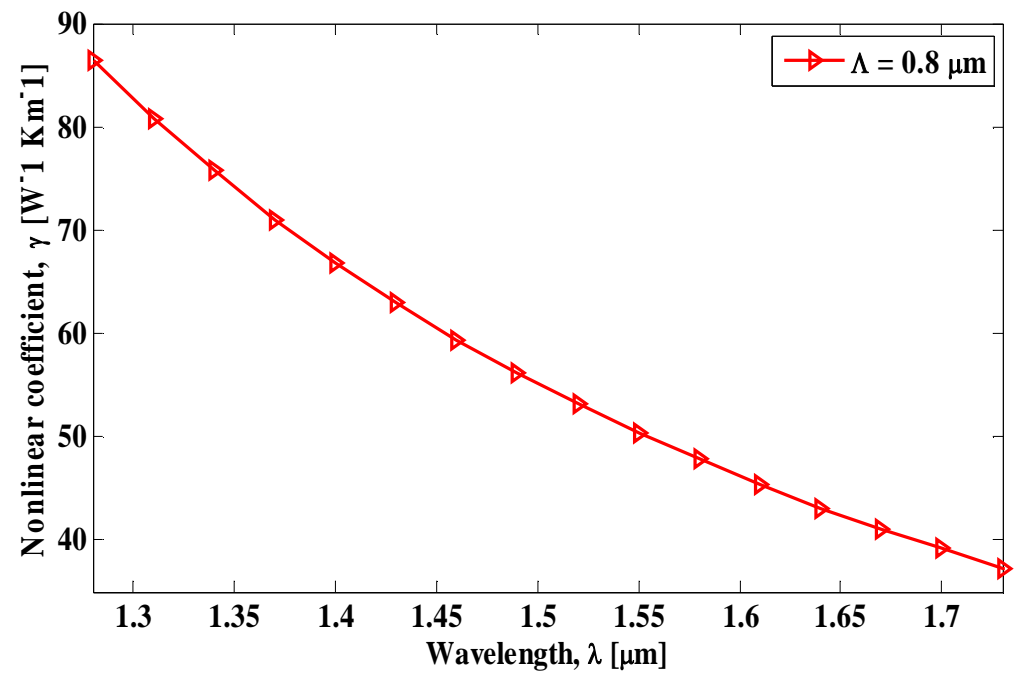

(b)

Figure 6. (a) Effective area versus wavelength curve and (b) Non-linear coefficient versus wavelength curve of proposed

$$
\text { HyDCF optimum pitch }=0.8 \mu \mathrm{m} \text {. }
$$

The observation of optimal and varied confinement loss with respect to wavelength for varying pitch from optimum value of the reported HyDCF are displayed on the figure 7 . From the plot $3.756 \times 10^{-10} \mathrm{~dB} / \mathrm{m}$ is observed as optimum value of confinement loss at $1550 \mathrm{~nm}$ wavelength. Varying the pitch value $0.8,0.9,1$ and $1.1 \mu \mathrm{m}$ the confinement loss changes $3.756 \times 10^{-10} \mathrm{~dB} / \mathrm{m}, 3.176 \times 10^{-10} \mathrm{~dB} / \mathrm{m}, 2.369 \times 10^{-10} \mathrm{~dB} / \mathrm{m}$ and $2.258 \times 10^{-10} \mathrm{~dB} / \mathrm{m}$, respectively. Confinement loss is a main drawback of a photonic crystal fiber [31]. From the curve it is evident that this modified hybrid fiber design expose very low confinement loss too. The design shows the confinement loss in the range of $10^{-10}$ range which is lower in other periodic structures referenced in the table. 


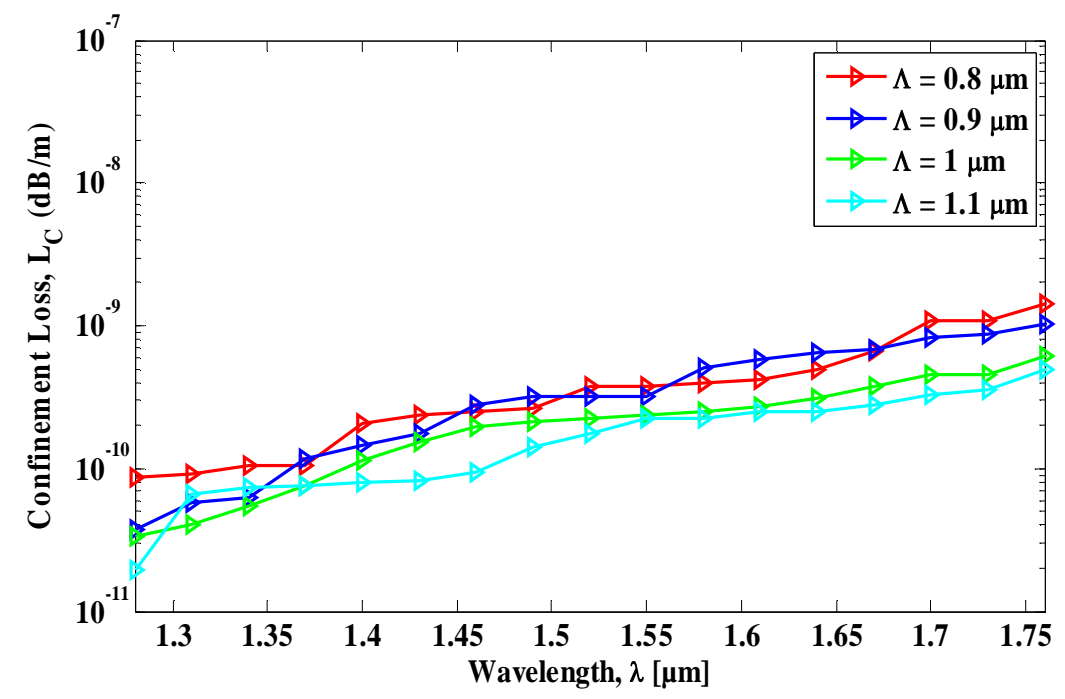

Figure 7. Confinement loss versus wavelength curve for optimum design parameters with variation of pitch.

The spreading of fundamental optical field for $\mathrm{x}$ and $\mathrm{y}$ polarization modes at the effective wavelength of $1550 \mathrm{~nm}$ has been revealed in figure 8(a) and (b). Conferring to simulation results it is evident that modes in $\mathrm{x}$ and $\mathrm{y}$ polarization are firmly bounded at the centre core area due to high availability of index in the centre than the cladding.

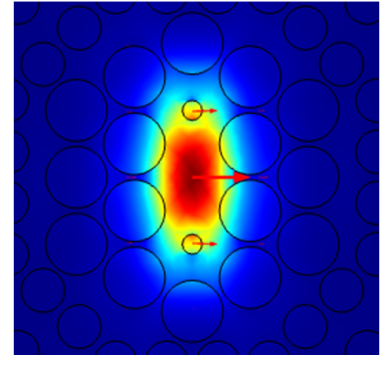

(a)

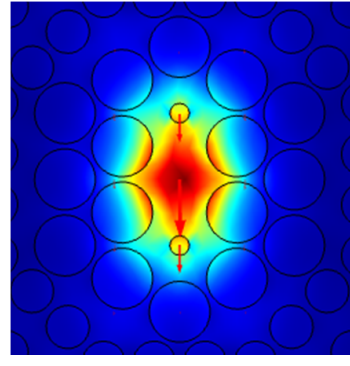

(b)

Figure 8. Field Fundamental field distribution modes for (a) x-polarization and (b) y-polarization at effective wavelength.

Table 1. Comparison of Optical Properties Between proposed HyDCF and other designs

\begin{tabular}{|c|c|c|c|}
\hline PCFs & $\begin{array}{c}\text { Dispersion, } \mathbf{D}(\boldsymbol{\lambda}) \\
{[\mathbf{p s} / \mathbf{( n m . k m )}}\end{array}$ & $\begin{array}{c}\text { Birefringence, } \\
\mathbf{B}=\left|\mathbf{n}_{\mathbf{x}}-\mathbf{n}_{\mathbf{y}}\right|\end{array}$ & $\begin{array}{c}\text { Confinement Loss, } \\
\mathbf{L}_{\mathbf{c}}[\mathbf{d B} / \mathbf{m}]\end{array}$ \\
\hline Ref. [12] & -588 & $1.81 \times 10^{-2}$ & $<10$ \\
\hline Ref. [13] & -239.5 & $1.67 \times 10^{-2}$ & $--0^{-5}$ \\
\hline Ref. [14] & -400 & $1.60 \times 10^{-2}$ & --- \\
\hline Ref. [16] & -331 & $2.75 \times 10^{-2}$ & 4.54 \\
\hline Ref. [17] & -294.1 & $2.13 \times 10^{-2}$ & $3 \times 10^{-6}$ \\
\hline Ref. [18] & -390 & $10^{-5}$ & $<10^{-2}$ \\
\hline Ref. [19] & -345 & $2.78 \times 10^{-2}$ & $3.756 \times 10^{-10}$ \\
\hline Proposed HyDCF & -606 & $3.76 \times 10^{-2}$ & \\
\hline
\end{tabular}


The matching of residual dispersion slope of the reported HyDCF has shown dispersion compensation over S, C and L bands of telecommunication is validated in the residual dispersion curve. The commercially available standard optical fibres faces the dispersion problem while transmitting signal into long distance. The dispersion compensating fibre allows to compensate the dispersion but there are some residue of dispersion while transmitting signal to long distance. This residual dispersion can be compensated by accomplishing single mode operation to certain distance here it is computed successfully through $50 \mathrm{~km}$ distance. Also commercially available standard fibers are not polarization maintaining fibres. This HyDCF microstructure photonic crystal fiber contains the high birefringence property which is suitable for sensing application and high nonlinearity property is the primary attribute of supercontinuum generation. The real-world usage of the proposed HyDCF will be significant in the field of communication applications and optical sensing. Lastly, an appraisal is prepared concerning birefringence and residual dispersion characteristics between the offered HyDCF and other fibres formerly studied for dispersion compensation and sensing applications. Table 1 displays the assessment of these optical characteristics.

\section{Conclusions}

The proposed artificially defected core hybrid photonic crystal fiber design concurrently demonstrates high birefringence and nonlinearity for use as an optical sensor. Dispersion coefficient in high negative value is achieved in this design for attaining dispersion compensating applications. The stated HyDCF achieve $3.76 \times 10^{-2}$ as high birefringence and highly nonlinear quantity of $50.34 \mathrm{~W}^{-1} \mathrm{~km}^{-1}$ at the effective wavelength of $1550 \mathrm{~nm}$ which recommends it as a good nominee for optical sensor. The simulation results also illustrate the negatively flattened residual dispersion of $-2.703 \pm 0.734 \mathrm{ps} /(\mathrm{nm} . \mathrm{km})$ within the $\mathrm{S}, \mathrm{C}$ and $\mathrm{L}$ telecommunication bands. The design possess low confinement loss which is very much important in photonic crystal fiber waveguide designs. The optical properties are very much suitable for endlessly single mode operation over the range of $1350-1800 \mathrm{~nm}$ wavelength. The confinement loss is obtained $3.756 \times 10$ ${ }^{10} \mathrm{~dB} / \mathrm{m}$ which is comparatively low than the other hybrid and complex structures of photonic crystal fiber. The excellent guiding properties lead this fiber design to be capable of other optical sensing utilization and wideband dispersion compensation in high bit stream rate in wavelength division multiplexing transmission systems.

Conflicts of Interest: The authors declare no conflict of interest.

\section{References}

1. $\quad$ Russell, Philip St J. "Photonic-crystal fibers." Journal of Lightwave Technology 24.12 (2006): 4729-4749.

2. Habib, M. Selim, et al. "Microstructure holey fibers as wideband dispersion compensating media for high speed transmission system." Optik 124.21 (2013):4984-4988.

3. L. Gruner-Nielsen, M. Wandel, P. Kristensen et al., "Dispersion-compensating fibers," Journal of Lightwave Technology, vol. 23, no. 11, pp. 3566-3579, Nov. 2005.

4. K. Saitoh, M. Koshiba, T. Hasegawa, and E. Sasaoka, "Chromatic dispersion control in photonic crystal fibers: application to ultra-flattened dispersion," Optics Express, vol. 11, no. 8, p. 843, Apr. 2003.

5. J. Laegsgaard, S. E. BarkouLibori, K. Hougaard et al., "Dispersion Properties of Photonic Crystal Fibers - Issues and Opportunities," MRS Proceedings, vol. 797, Jan. 2003.

6. L. Grüner-Nielsen, S. N. Knudsen, B. Edvold et al., "Dispersion Compensating Fibers," Optical Fiber Technology, vol. 6, no. 2, pp. 164-180, Apr. 2000.

7. S.G. Li, X. D. Liu, L. T. Hou, "Numerical study on dispersion compensating property in photonic crystal fibers," Acta Phys. Sin., (2004) 1880.

8. X. Zhao, G. Zhou, S. Li et al., "Photonic crystal fiber for dispersion compensation," Applied Optics, vol. 47, no. 28, p. 5190 , Sep. 2008.

9. T. Zhong-Wei, N. Ti-Gang, L. Yan, T. Zhi, and J. Shui-Sheng, "Suppression of the interactions between fiber gratings used as dispersion compensators in dense wavelength-division multiplexing systems," Chinese Physics, vol. 15, no. 8, pp. 1819-1823, Jul. 2006.

10. Y. Ni, L. Zhang, L. An, J. Peng and C. C. Fan, "Dual- core photonic crystal fiber for dispersion compensation," IEEE Photon. Technol.Lett. (2004) 1516.

11. S. F. Kaijage, Y. Namihira, N. H. Hai, F. Begum, S. M. A. Razzak, T. Kinjo, K. Miyagi, and N. Zou, "Broadband 
Dispersion Compensating Octagonal Photonic Crystal Fibre for Optical Communication Applications," Japanese Journal of Applied Physics, vol. 48, no. 5, p. 052401, May 2009.

12. M. Selim Habib, M. Samiul Habib, S. M. Abdur Razzak, and M. Anwar Hossain, "Proposal for highly birefringent broadband dispersion compensating octagonal photonic crystal fiber," Optical Fibre Technology, vol. 19, no. 5, pp. 461-467, Oct. 2013.

13. Kaijage, Shubi F., Yoshinori Namihira, Nguyen H. Hai, Feroza Begum, SM Abdur Razzak, Tatsuya Kinjo, Kazuya Miyagi, and Nianyu Zou. "Broadband dispersion compensating octagonal photonic crystal fiber for optical communication applications." Japanese Journal of Applied Physics 48, no. 5R (2009): 052401.

14. A. Agrawal, N. Kejalakshmy, J. Chen, B. M. A. Rahman, and K. T. V. Grattan, "Golden spiral photonic crystal fiber: polarization and dispersion properties," Optics Letters, vol. 33, no. 22, p. 2716, Nov. 2008.

15. A. Halder, "Highly birefringent photonic crystal fiber for dispersion compensation over $\mathrm{E}+\mathrm{S}+\mathrm{C}+\mathrm{L}$ communication bands," 2016 5th International Conference on Informatics, Electronics and Vision (ICIEV), May 2016.

16. M. S. Ali, K. M. Nasim, R. Ahmad, M. A. G. Khan, and M. S. Habib, "A defected core highly birefringent dispersion compensating photonic crystal fiber,” 2013 2nd International Conference on Advances in Electrical Engineering (ICAEE), Dec. 2013.

17. M. S. Habib, M. S. Rana, M. Moniruzzaman, M. S. Ali, and N. Ahmed, "Highly birefringent broadbanddispersion-compensating photonic crystal fiber over the $\mathrm{E}+\mathrm{S}+\mathrm{C}+\mathrm{L}+\mathrm{U}$ wavelength bands," Optical Fibre Technology, vol. 20, no. 5, pp. 527-532, Oct. 2014

18. M. A. Islam, R. Ahmad, M. S. Ali, and K. M. Nasim, "Proposal for highly residual dispersion compensating defected core decagonal photonic crystal fiber over $\mathrm{S}+\mathrm{C}+\mathrm{L}+\mathrm{U}$ wavelength bands," Optical Engineering, vol. 53, no. 7, p. 076106, Jul. 2014.

19. S. Ali, N. Ahmed, M. Islam, S. A. Aljunid et al., "A high birefringent PCF with RDS matched dispersion compensation over S+C+L communication bands," 2016 3rd International Conference on Electronic Design (ICED), Aug. 2016.

20. N. A. Issa, M. A. van Eijkelenborg, M. Fellew et al., "Fabrication and study of microstructured optical fibres with elliptical holes," Optics Letters, vol. 29, no. 12, p. 1336, Jun. 2004.

21. F. Quiñónez, J. W. Menezes, L. Cescato, V. F. Rodriguez-Esquerre, H. Hernandez-Figueroa, and R. D. Mansano, "Band gap of hexagonal 2D photonic crystals with elliptical holes recorded by interference lithography," Optics Express, vol. 14, no. 11, p. 4873, 2006.

22. R. T. Bise and D. J. Trevor, "Sol-gel derived microstructured fiber: fabrication and characterization," OFC/NFOEC Technical Digest. Optical Fibre Communication Conference, 2005.

23. K. Saitoh and M. Koshiba, "Full-vectorial imaginary-distance beam propagation method based on a finite element scheme: application to photonic crystal fibres," IEEE Journal of Quantum Electronics, vol. 38, no. 7, pp. 927-933, Jul. 2002.

24. Y. Dong, X. Bao, and L. Chen, "Distributed temperature sensing based on birefringence effect on transient Brillouin grating in a polarization-maintaining photonic crystal fiber,” Optics Letters, vol. 34, no. 17, p.2590, Aug. 2009.

25. S. F. Kaijage, Y. Namihira, N. H. Hai et al., "Multiple defect-core hexagonal photonic crystal fiber with flattened dispersion and polarization maintaining properties," Optical Review, vol. 15, no. 1, pp. 31-37, Jan. 2008.

26. T. A. Birks, D. Mogilevtsev, J. C. Knight, and P. St. J. Russell, "Dispersion compensation using single material fibres," IEEE Photonics Technology Letters, vol. 11, no. 6, pp. 674-676, Jun. 1999.

27. M. S. Habib, M. S. Habib, M. I. Hasan, and S. M. A. Razzak, "Tailoring polarization maintaining broadband residual dispersion compensating octagonal photonic crystal fibres,” Optical Engineering, vol. 52, no. 11, p. 116111, Nov. 2013.

28. A. W. Snyder and J. D. Love, "Optical Waveguide Theory,” 1984.

29. T. A. Birks, J. C. Knight, and P. S. J. Russell, "Endlessly single-mode photonic crystal fiber," Optics Letters, vol. 22, no. 13, p. 961, Jul. 1997.

30. Ju, Jian, Wei Jin, and M. S. Demokan. "Properties of a highly birefringent photonic crystal fiber." IEEE photonics technology letters 15.10 (2003): 1375-1377.

31. Napierala, Marek, et al. "Photonic crystal fiber with large mode area and characteristic bending properties." IEEE Photonics Technology Letters 24.16 (2012): 1409-1411.

32. Niels Asger Mortensen, "Effective area of photonic crystal fibers", Opt. Express 10 (2002) 341-348. 
33. G.S. Wiederhecker, C.M.B. Cordeiro, F. Couny, F. Benabid, S.A. Maier, J.C. Knight, C.H.B. Cruz, H.L. Fragnito, Field enhancement within an optical fibre with a subwavelength air core, Nat. Photon. 1 (2) (2007) 115-118.

34. Y. Ruan, H. Ebendorff-Heidepriem, V. Afshar, T.M. Monro, Light confinement within nanoholes in nanostructured optical fibers, Opt. Exp. 18 (December (25)) (2010) 26018-26026.

35. M.F.H. Arif, M.J.H. Biddut, Enhancement of relative sensitivity of photonic crystal fiber with high birefringence and low confinement loss, Optik Int. J. Light Electron Opt. 131 (Feb. 2017) 697e704.

36. Ahmed, Kawsar, Md Shadidul Islam, and Bikash Kumar Paul. "Design and numerical analysis: effect of core and cladding area on hybrid hexagonal microstructure optical fiber in environment pollution sensing applications." Karbala International Journal of Modern Science 3.1 (2017): 29-38. 